\title{
Betatron coupling: Merging Hamiltonian and matrix approaches
}

\author{
R. Calaga and R. Tomás \\ BNL, Upton, New York 11973, USA
}

\section{A. Franchi}

GSI, Planckstrasse 1, 64291 Darmstadt, Germany

(Received 7 December 2004; published 9 March 2005)

\begin{abstract}
Betatron coupling is usually analyzed using either matrix formalism or Hamiltonian perturbation theory. The latter is less exact but provides a better physical insight. In this paper direct relations are derived between the two formalisms. This makes it possible to interpret the matrix approach in terms of resonances, as well as use results of both formalisms indistinctly. An approach to measure the complete coupling matrix and its determinant from turn-by-turn data is presented. Simulations using methodical accelerator design MAD-X, an accelerator design and tracking program, were performed to validate the relations and understand the scope of their application to real accelerators such as the Relativistic Heavy Ion Collider.
\end{abstract}

DOI: 10.1103/PhysRevSTAB.8.034001

PACS numbers: 29.27.Bd

\section{INTRODUCTION}

Betatron coupling in circular accelerators has been widely studied using both matrix formalism and Hamiltonian perturbation theory. In the matrix formalism the transverse beam motion is parametrized by a $4 \times 4$ one-turn matrix factorized into a block diagonal normal mode form consisting of the respective Twiss functions and a $2 \times 2$ coupling matrix ( $\mathbf{C}$ matrix). In the Hamiltonian approach the coupling elements are considered to be a perturbation to the uncoupled lattice. The equations of motion are expanded up to the first order in those perturbations. In this paper, direct relations between the two approaches are derived and discussed in detail.

Numerical methods such as Fourier transform and singular value decomposition (SVD) have been demonstrated to be effective in measuring relevant quantities in both formalisms [1-4]. However, in the matrix formalism, measurements have been constrained to three of the four elements in the coupling matrix [2,3]. The individual elements of the coupling matrix usually have complicated behavior around the ring. One often has to rely on fitting techniques to identify sources of local coupling [2].

An approach to extend the existing methods to measure the complete $\mathbf{C}$ matrix and hence the determinant is presented. The behavior of the determinant in the presence of coupling sources is discussed in detail and its advantage of localizing these coupling sources is transparent. The applicability of the expression relating closest tune approach $\left(\Delta Q_{\text {min }}\right)$ and $\overline{\mathbf{C}}$ matrix given in $[5,6]$ is also studied.

\section{HAMILTONIAN TERMS AND COUPLING MATRIX}

To relate the coupling matrix to the Hamiltonian terms the expressions describing the turn-by-turn motion from both formalisms are compared. This is achieved by intro- ducing the canonical momentum in the matrix framework and constructing a complex variable. Prior to this the two approaches are briefly introduced.

\section{A. Resonance driving terms}

Using Hamiltonian and normal form theory a weakly coupled lattice is treated as a perturbation to the uncoupled lattice. The turn-by-turn normalized particle positions and momenta at a location $s$ are described as [7]

$$
\begin{aligned}
\hat{x}-i \hat{p}_{x}^{h}= & \sqrt{2 I_{x}} e^{i \psi_{x}}-2 i f_{1001} \sqrt{2 I_{y}} e^{i \psi_{y}} \\
& -2 i f_{1010} \sqrt{2 I_{y}} e^{-i \psi_{y},} \\
\hat{y}-i \hat{p}_{y}^{h}= & \sqrt{2 I_{y}} e^{i \psi_{y}}-2 i f_{1001}^{*} \sqrt{2 I_{x}} e^{i \psi_{x}} \\
& -2 i f_{1010} \sqrt{2 I_{x}} e^{-i \psi_{x}},
\end{aligned}
$$

where $I_{x, y}$ are the horizontal and the vertical invariants. The phases of the oscillations $\psi_{x, y}$ are expressed as a function of the tunes $Q_{x, y}$, the turn number $N$ and the initial phases $\phi_{x 0, y 0}$ as $\psi_{x, y}=2 \pi Q_{x, y} N+\phi_{x 0, y 0}$. The resonance driving terms (RDTs) $f_{1001}$ and $f_{1010}$ are proportional to the Hamiltonian terms [7] and drive the difference and the sum resonances, respectively. These terms are functions of the uncoupled lattice parameters at the location of both the coupling elements and the observation point $s$ given by

$$
f(s)_{10010}=-\frac{1}{4\left(1-e^{2 \pi i\left(Q_{x} \mp Q_{y}\right)}\right)} \sum_{l} k_{l} \sqrt{\beta_{x}^{l} \beta_{y}^{l}} e^{i\left(\Delta \phi_{x}^{s l}+\Delta \phi_{y}^{s l}\right)},
$$

where $k_{l}$ is the $l$ th integrated skew quadrupole strength, $\beta_{x, y}^{l}$ are the Twiss functions at the location of the $l$ th skew quadrupole, $\Delta \phi_{x, y}^{s l}$ are the phase advances between the 
observation point $s$ and the $l$ th skew quadrupole, and $Q_{x, y}$ are the horizontal and vertical tunes.

\section{B. Matrix formalism}

In the matrix approach, the coupled motion [8] is parametrized by factoring the one-turn matrix into block diagonal normal mode form by means of the similarity transformation given by the symplectic $4 \times 4$ matrix $\mathbf{V}$, of the form

$$
\mathbf{V}=\left(\begin{array}{cc}
\gamma \mathbf{I} & \mathbf{C} \\
-\mathbf{C}^{+} & \gamma \mathbf{I}
\end{array}\right)
$$

where $\mathbf{I}$ is the $2 \times 2$ identity matrix, $\mathbf{C}$ is the $2 \times 2$ coupling matrix, and $\mathbf{C}^{+}$is its symplectic conjugate requiring $|\mathbf{C}|+\gamma^{2}=1$. By means of another similarity transformation, the $\beta$ dependence is normalized out of $\mathbf{C}$,

$$
\overline{\mathbf{C}}=\mathbf{G}_{\mathbf{a}} \mathbf{C G}_{\mathbf{b}}^{-1},
$$

where

$$
\mathbf{G}_{\mathbf{a}, \mathbf{b}}=\left[\begin{array}{cc}
\frac{1}{\sqrt{\beta_{a, b}}} & 0 \\
\frac{\alpha_{a, b}}{\sqrt{\beta_{a, b}}} & \sqrt{\beta_{a, b}}
\end{array}\right]
$$

are the normalization matrices for the $a$ and $b$ modes [9]. Note that $|\overline{\mathbf{C}}|=|\mathbf{C}|$. The normalized motion in the horizontal and vertical planes is given by [9]

$$
\left(\begin{array}{c}
\hat{x} \\
\hat{p}_{x} \\
\hat{y} \\
\hat{p}_{y}
\end{array}\right)=\left(\begin{array}{cccc}
\gamma & 0 & \bar{C}_{11} & \bar{C}_{12} \\
0 & \gamma & \bar{C}_{21} & \bar{C}_{22} \\
-\bar{C}_{22} & \bar{C}_{12} & \gamma & 0 \\
\bar{C}_{21} & -\bar{C}_{11} & 0 & \gamma
\end{array}\right)\left(\begin{array}{c}
A_{x} \cos \psi_{x} \\
A_{x} \sin \psi_{x} \\
A_{y} \cos \psi_{y} \\
A_{y} \sin \psi_{y}
\end{array}\right) .
$$

Using the above expressions for normalized positions and momenta, the complex variables are given by

$$
\begin{aligned}
\hat{x}+i \hat{p}_{x}= & \gamma A_{x} e^{i \psi_{x}}+\frac{A_{y}}{2}\left[\left(\bar{C}_{11}+i \bar{C}_{12}+i \bar{C}_{21}-\bar{C}_{22}\right) e^{-i \psi_{y}}\right. \\
& \left.+\left(\bar{C}_{11}-i \bar{C}_{12}+i \bar{C}_{21}+\bar{C}_{22}\right) e^{i \psi_{y}}\right], \\
\hat{y}+i \hat{p}_{y}= & \gamma A_{y} e^{i \psi_{y}}+\frac{A_{x}}{2}\left[\left(\bar{C}_{11}+i \bar{C}_{12}+i \bar{C}_{21}-\bar{C}_{22}\right) e^{-i \psi_{x}}\right. \\
& \left.+\left(-\bar{C}_{11}-i \bar{C}_{12}+i \bar{C}_{21}-\bar{C}_{22}\right) e^{i \psi_{x}}\right] .
\end{aligned}
$$

Note that the convention for momenta used in Hamiltonian theory described in [7] is the negative of that used in matrix formalism described in [10] $\left(\hat{p}_{x}^{h}=-\hat{p}_{x}\right)$.

\section{Relating the $\mathbf{C}$ matrix to RDTs}

The relation to the Hamiltonian formalism can now be established by directly comparing Eqs. (1) and (2) to Eqs. (7) and (8) obtaining

$$
\begin{gathered}
f_{1001}=\frac{1}{4 \gamma}\left(\bar{C}_{12}-\bar{C}_{21}+i \bar{C}_{11}+i \bar{C}_{22}\right), \\
f_{1010}=\frac{1}{4 \gamma}\left(-\bar{C}_{12}-\bar{C}_{21}+i \bar{C}_{11}-i \bar{C}_{22}\right),
\end{gathered}
$$

or, equivalently expressing $\overline{\mathbf{C}}$ as a function of the RDTs,

$$
\begin{gathered}
\frac{1}{2 \gamma} \bar{C}_{12}=-\operatorname{Re}\left\{f_{1010} \mp f_{1001}\right\}, \\
\frac{1}{2 \gamma} \bar{C}_{112}=\operatorname{Im}\left\{f_{1001} \pm f_{1010}\right\},
\end{gathered}
$$

where $\operatorname{Re}$ and Im stand for real and imaginary parts, respectively. The determinant of $\overline{\mathbf{C}}$ can also be related to the RDTs as

$$
\frac{|\overline{\mathbf{C}}|}{4 \gamma^{2}}=\left|f_{1001}\right|^{2}-\left|f_{1010}\right|^{2},
$$

and using $|\overline{\mathbf{C}}|+\gamma^{2}=1$ yields

$$
\begin{gathered}
|\overline{\mathbf{C}}|=1-\frac{1}{1+4\left(\left|f_{1001}\right|^{2}-\left|f_{1010}\right|^{2}\right)}, \\
\gamma^{2}=\frac{1}{1+4\left(\left|f_{1001}\right|^{2}-\left|f_{1010}\right|^{2}\right)} .
\end{gathered}
$$

These expressions have a direct interpretation: If $|\overline{\mathbf{C}}|$ is positive the difference resonance $\left(f_{1001}\right)$ dominates; and if it is negative the sum resonance $\left(f_{1010}\right)$ dominates. From these expressions it is also observed that a null $|\overline{\mathbf{C}}|$ does not imply null coupling, but $\left|f_{1001}\right|=\left|f_{1010}\right|$. If $\left|f_{1010}\right|^{2}>$ $\left(\frac{1}{4}+\left|f_{1001}\right|\right)$, then $\gamma^{2}<0$ and the particle motion is unstable [see discussion in [9] after Eq. (12)].

In [4] it was demonstrated that the amplitude of the RDTs remains constant along sections free of coupling sources and experiences abrupt jumps at locations with couplers. In [4] it is also shown that the relative longitudinal variations of the RDTs become smaller as the tunes approach the resonance. On the resonance, the amplitude of the RDTs becomes invariant around the ring. Thus, by virtue of Eq. (13), the determinant of $\overline{\mathbf{C}}$ also tends to be invariant around the ring as the tunes approach the resonance.

\section{Simulations}

To confirm the relations derived above, simulations were carried out with the aid of MAD-X [11]. A simple ring consisting of 80 FODO cells is constructed using parameters shown in Table I. Three skew quadrupoles of different strengths were placed at arbitrary locations to introduce transverse coupling in the lattice. 
TABLE I. FODO lattice parameters.

\begin{tabular}{lcc}
\hline \hline \multicolumn{1}{c}{ Species } & Length $(\mathrm{m})$ & Strength \\
\hline Dipoles & 6.5 & $0.039 \mathrm{rad}$ \\
Quadrupoles & 0.5 & $0.1 \mathrm{~m}^{-2}$ \\
Skew quadrupoles & 0.2 & $4.3 \times 10^{-3} \mathrm{~m}^{-2}$ \\
Drifts & 0.25 & $\cdots$ \\
\hline \hline
\end{tabular}

\section{1. $f_{1001}$ and $\overline{\mathbf{C}}$ matrix}

Twiss functions and coupling matrix elements in the form of $\mathbf{R}$ matrix (Edwards-Teng parametrization [8]) are directly available from MAD-X. The $\mathbf{C}$ matrix is determined by a simple transformation of the $\mathbf{R}$ matrix given by

$$
\mathbf{C}=\frac{1}{1+|\mathbf{R}|} \mathbf{J}^{-\mathbf{1}} \mathbf{R} \mathbf{J}
$$

where

$$
\mathbf{J}=\left[\begin{array}{cc}
0 & 1 \\
-1 & 0
\end{array}\right]
$$

$\overline{\mathbf{C}}$ is obtained from Eq. (5) by normalizing out the beta dependence from $\mathbf{C}$. To calculate the RDTs $f_{1001}$ and $f_{1010}$ from MAD-X, the first order approximation given by Eq. (3) is used. Figure 1 shows a comparison between the RDTs and $\overline{\mathbf{C}}$ matrix elements as derived in Eqs. (9) and (13). The rms of the differences between the compared quantities are smaller than $10^{-6}$, thus numerically validating the relations.

\section{Dependence on skew quadrupole strengths}

The relations between $|\overline{\mathbf{C}}|$ and the RDTs are first order approximations. To investigate the accuracy of these relations, the mean of the ratio of the quantities compared in Fig. 1 is computed for increasing skew quadrupole strengths. The horizontal and vertical tunes are fixed at $Q_{x}=18.226$ and $Q_{y}=17.232$, respectively. Figure 2 shows a plot of this mean ratio along with the standard deviation of the ratio as a function of closest tune approach $\left(\Delta Q_{\min }\right)$ produced by the three skew quadrupoles. For this particular case, the quantities compared agree in the percent level for a $\Delta Q_{\min }$ lower than $3 \times 10^{-3}$.

\section{Stop-band limits}

To explore the behavior of Eq. (13), a scan of horizontal tune $\left(Q_{x}\right)$ is performed with the vertical tune fixed at $Q_{y}=$ 0.228 . Figure 3 shows the driving terms $\left|f_{1001}\right|^{2}$ and $\left|f_{1010}\right|^{2}$ as well as $|\overline{\mathbf{C}}| / \gamma^{2}$ plotted as a function $Q_{x}$ for the FODO lattice described above. The dominance of $f_{1001}$ or $f_{1010}$ is seen in Fig. 3 depending on the proximity to either the difference or sum resonance, respectively. This transition is also evident from the behavior of $|\overline{\mathbf{C}}| / \gamma^{2}$ as it switches sign when we move from difference to the sum resonance in accordance with Eq. (13). There are missing data points at $Q_{x}=0.5$ due to the $1 / 2$ integer resonance.

\section{DETERMINANT OF $\overline{\mathrm{C}}$}

It has been demonstrated that $\bar{C}_{12} / \gamma, \bar{C}_{11} / \gamma, \bar{C}_{22} / \gamma$ are measured using turn-by-turn data from beam posi-

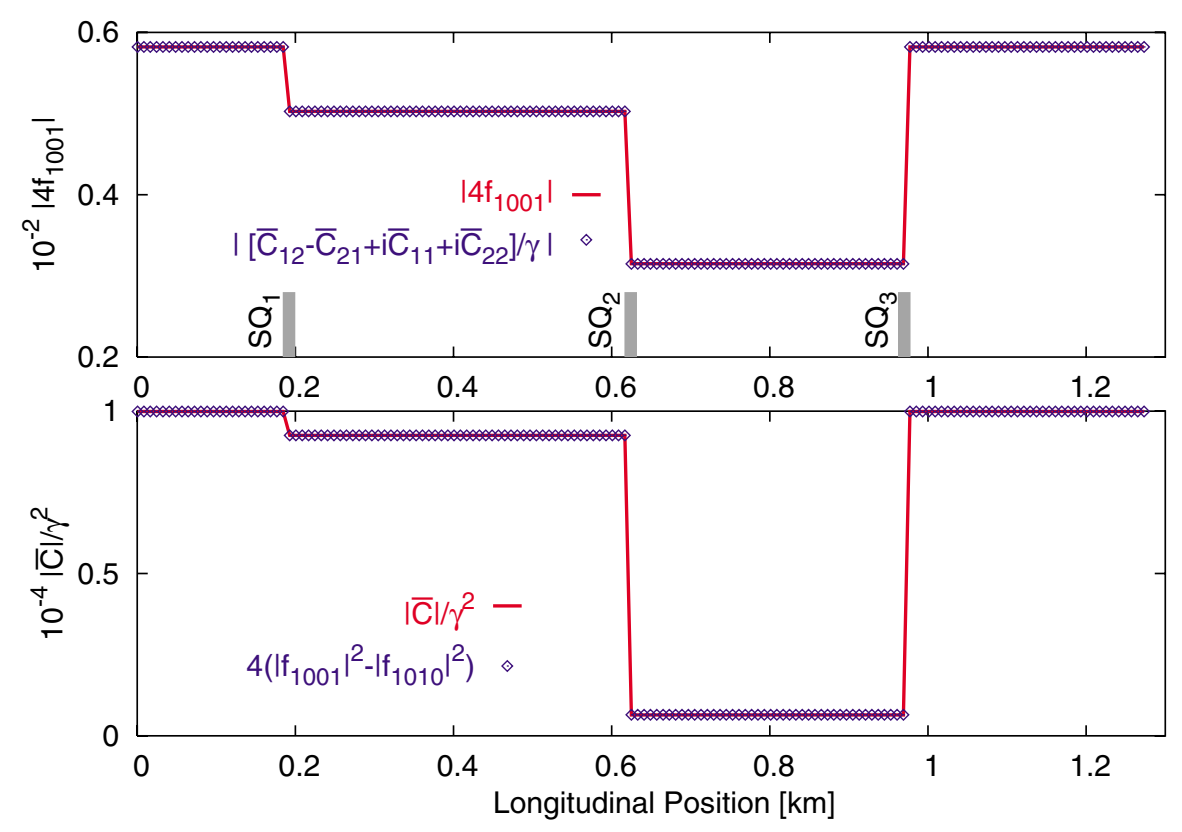

FIG. 1. (Color) Top: $4\left|f_{1001}\right|$ and $\left|\left[\left(\bar{C}_{12}-\bar{C}_{21}\right)+i\left(\bar{C}_{11}+\bar{C}_{22}\right)\right] / \gamma\right|$ plotted as a function of the longitudinal position. Bottom: $|\overline{\mathbf{C}}| / \gamma^{2}$ and $4\left(\left|f_{1001}\right|^{2}-\left|f_{1010}\right|^{2}\right)$ plotted as a function of longitudinal position. Horizontal and vertical tunes are $Q_{x}=18.226$ and $Q_{y}=$ 17.257, respectively. 


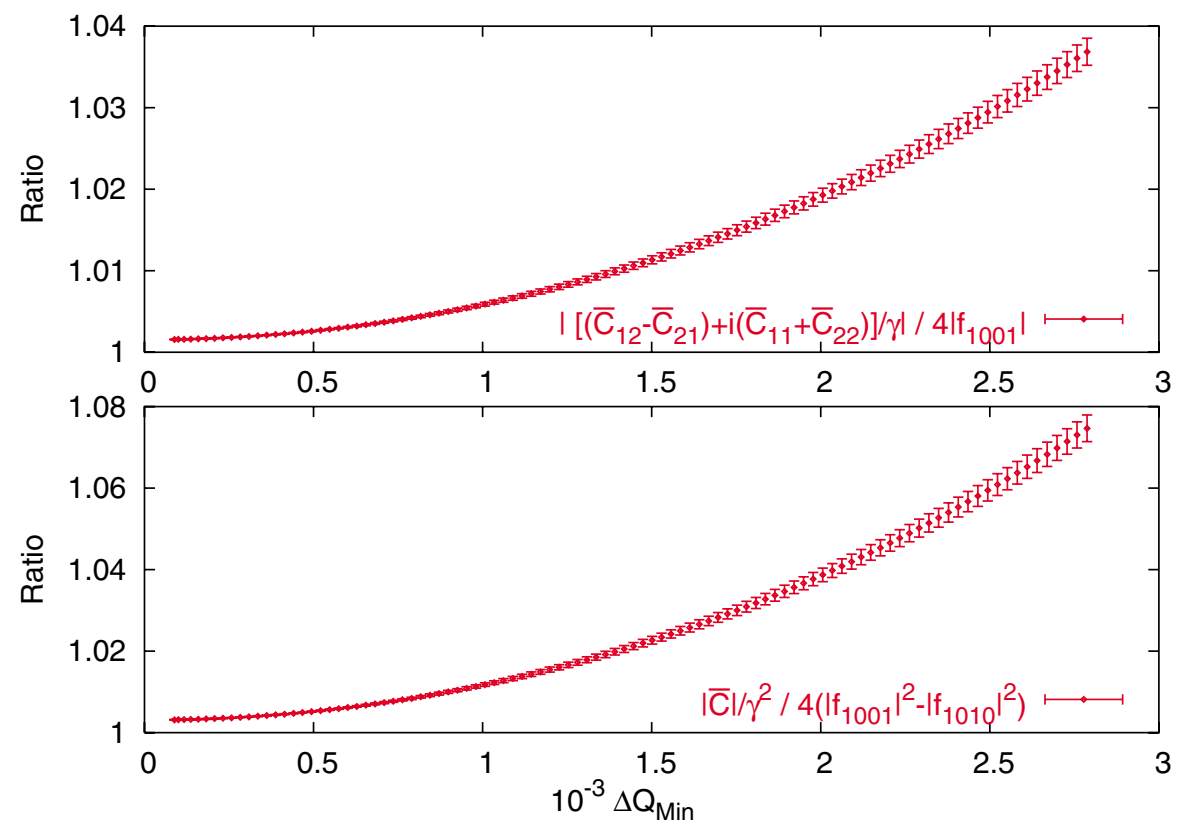

FIG. 2. (Color) Top: Mean of the ratio of $\left|\left[\left(\bar{C}_{12}-\bar{C}_{21}\right)+i\left(\bar{C}_{11}+\bar{C}_{22}\right)\right] / \gamma\right|$ and $4\left|f_{1001}\right|$. Bottom: Mean of the ratio of $|\overline{\mathbf{C}}| / \gamma^{2}$ and $4\left(\left|f_{1001}\right|^{2}-\left|f_{1010}\right|^{2}\right)$. The error bars represent the standard deviation of the ratio of the respective quantities. The horizontal and vertical tunes are $Q_{x}=18.226$ and $Q_{y}=17.232$.

tion monitors (BPMs) [2,3]. From $\bar{C}_{12} / \gamma$, one relies on fitting techniques to determine the location of coupler and estimate its strength to correct local coupling $[2,10]$. However, the $|\overline{\mathbf{C}}|$ like the RDTs is a constant in coupler free regions and exhibit abrupt jumps at the location of a coupler. These discontinuities are intuitive and can be identified simply by visual inspection to the nearest BPM, as seen in Fig. 1. The $|\overline{\mathbf{C}}|$ also allows one to easily estimate global quantities like $\Delta Q_{\min }$ and local quantities like the strength and polarity of the coupler which are useful during machine operation. However, it will be seen that $\bar{C}_{12} / \gamma$ has a better signal-to-noise ratio. Additionally, it has better resolution to locate an isolated coupler more accurately using appropriate fitting techniques, while $|\overline{\mathbf{C}}|$ has a resolution to within a region between the two nearest BPMs.

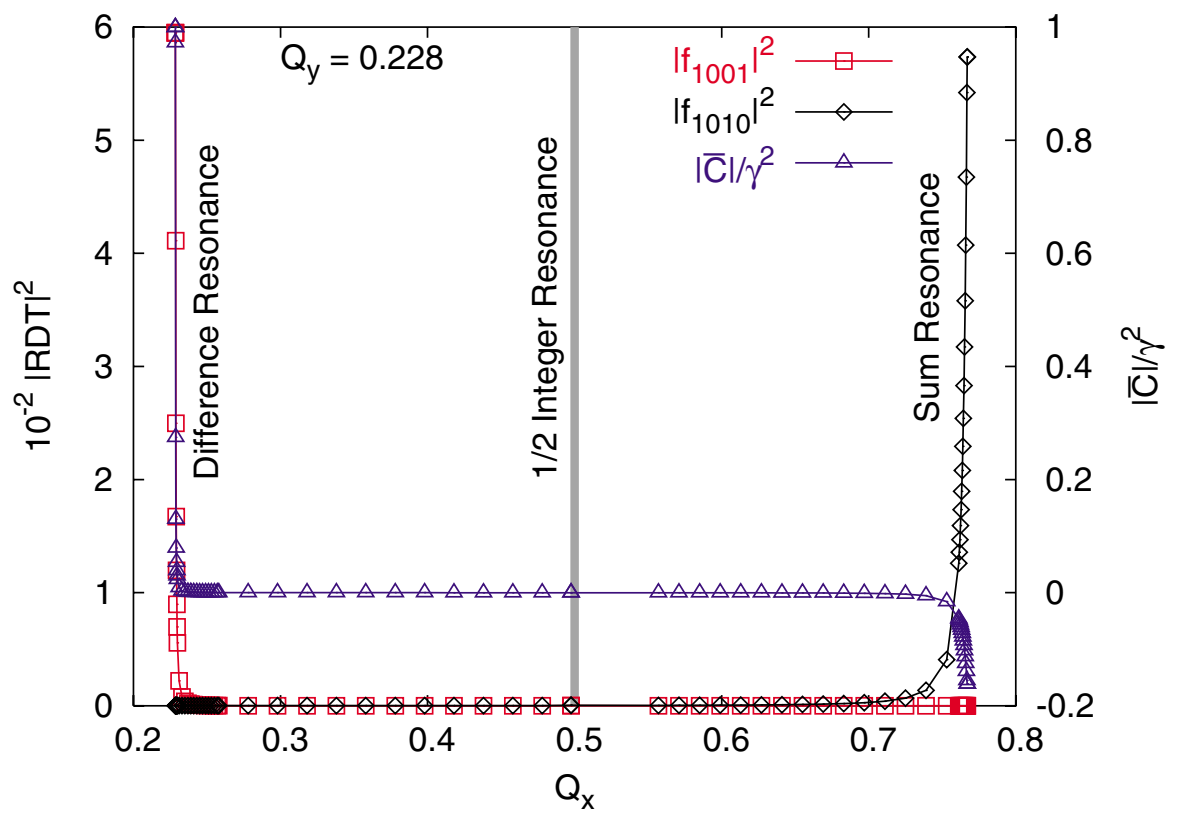

FIG. 3. (Color) $\left|f_{1001}\right|^{2},\left|f_{1010}\right|^{2}$, and $|\overline{\mathbf{C}}| / \gamma^{2}$ plotted as a function of $Q_{x}\left(Q_{y}=0.228\right)$. The stop bands at the sum and the difference resonance show the dramatic increase in these functions. The $1 / 2$ integer resonance line is plotted as a reference. 
To calculate the determinant, we propose a method to calculate $\bar{C}_{21}$ given $\bar{C}_{12}, \bar{C}_{11}$, and $\bar{C}_{22}$ at two locations with an arbitrary phase advance in both normal modes. Given two locations (1) and (2) in a coupler free region, $\bar{C}_{21}^{(1)}$ is given (see Sec. 1 in the appendix) by

$$
\begin{aligned}
\bar{C}_{21}^{(1)}= & \left(-\bar{C}_{11}^{(1)} \cos \phi_{a} \sin \phi_{b}+\bar{C}_{12}^{(1)} \cos \phi_{a} \sin \phi_{b}\right. \\
& \left.+\bar{C}_{22}^{(1)} \sin \phi_{a} \cos \phi_{b}-\bar{C}_{12}^{(2)}\right) /\left(\sin \phi_{a} \sin \phi_{b}\right),
\end{aligned}
$$

where $\phi_{a}$ and $\phi_{b}$ are the phase advances between the two observation locations of the normal modes. We will demonstrate the effectiveness of such a calculation in a simple FODO lattice as well as in a complicated lattice such as RHIC where the coupling sources are mainly localized in the interaction regions with little or no coupling in the arcs.

It is also important to note that RDTs calculated using fast-Fourier-transform techniques in [4] can be extended to compute $\overline{\mathbf{C}}$ matrix and hence the determinant according to Eqs. (11)-(15). The approach of $N$-turn map presented in [6] could also be extended to calculate both $\gamma$ and $|\overline{\mathbf{C}}|$.

\section{A. Calculation of $\bar{C}_{12} / \gamma$ using SVD}

For the purpose of this paper, we use an SVD-based method to construct the physical modes of beam $[3,12]$. We will briefly introduce this method to show how the $\overline{\mathbf{C}}$ matrix and its determinant are computed. A data matrix $B_{t \times m}$ consisting of $t$ columns of turn-by-turn data at the $m$ th BPM is decomposed using SVD [13] given by

$$
\mathbf{B}=\mathbf{U} \boldsymbol{\sigma} \mathbf{V}^{T}=\sum_{\mathrm{i}} \sigma_{i} u_{i} v_{i}^{T},
$$

where $\Sigma$ is a diagonal matrix of singular values $\sigma_{i}$ with $\sigma_{1} \geq \sigma_{2} \geq \cdots \geq \sigma_{n} \geq 0 . U_{t \times t}$ and $V_{m \times m}$ are normalized eigenvectors of $A A^{T}$ and $A^{T} A$ representing the temporal and spatial patterns of the leading singular values.

In the presence of coupling, the physical modes of beam motion are related to the SVD modes by a $4 \times 4$ rotation matrix $O$ [12]. The normal modes are reconstructed from BPM data of either horizontal or vertical planes with the knowledge of the $O$ matrix. From the horizontal plane, $\bar{C}_{12} / \gamma$ is given by [3]

$$
\frac{\bar{C}_{12}}{\gamma}=\operatorname{sgn}\left(\sin \Delta \psi_{a}\right) \sqrt{\frac{\tilde{A}_{a} \tilde{A}_{b}}{A_{a} A_{b}} \sin \Delta \psi_{a} \sin \Delta \psi_{b}}
$$

Here $A_{a, b}^{2} \equiv \bar{J}_{a, b} \beta_{a, b} \gamma^{2}$ and $\tilde{A}_{a, b}^{2} \equiv \bar{J}_{a, b} \beta_{b, a} c_{a, b}^{2}$, where $\bar{J}_{a, b}$ are the average actions and $\beta_{a, b}$ are the beta functions for the two normal modes. $\gamma=\sqrt{1-\mid \bar{C}} \mid, \quad c_{b}=$ $\sqrt{\bar{C}_{11}^{2}+\bar{C}_{12}^{2}}$, and $\Delta \psi_{b}=\arctan \left(\bar{C}_{12} / \bar{C}_{11}\right)$. The vertical plane is expressed by replacing $x$ to $y$ and $a$ to $b$, and with $c_{a}=\sqrt{\bar{C}_{22}^{2}+\bar{C}_{12}^{2}}$ and $\Delta \psi_{a}=-\arctan \left(\bar{C}_{12} / \bar{C}_{22}\right)$. Therefore,

$$
\begin{gathered}
\frac{\bar{C}_{11}}{\gamma}=\frac{\bar{C}_{12}}{\gamma} \cot \Delta \psi_{b}, \\
\frac{\bar{C}_{22}}{\gamma}=-\frac{\bar{C}_{12}}{\gamma} \cot \Delta \psi_{a} .
\end{gathered}
$$

\section{B. Calculation of $|\bar{C}| / \gamma^{2}$ from tracking data}

Using Eqs. (17) and (19)-(21), $|\overline{\mathbf{C}}| / \gamma^{2}$ can now be calculated from turn-by-turn BPM data. Single-particle tracking is performed using MAD-X on the FODO lattice described earlier to generate turn-by-turn data at a large number of BPM locations. For all simulations, we assume dual-plane BPMs capable of measuring transverse positions in both planes, unless otherwise mentioned. The location of BPMs (1) and (2) in Eq. (17) to calculate $\bar{C}_{21} / \gamma$ is arbitrary and only requires that there are no coupling sources present between them. For the purpose of the simulations BPM (2) is chosen to be the next upstream detector from location (1). Figure 4 shows a comparison of MAD-X model and SVD computed values of $|\overline{\mathbf{C}}| / \gamma^{2}$. The overall discrepancy is smaller than $0.01 \%$ and increases up to $1 \%$ in the coupler regions. To minimize the discrepancy in the coupler region, $\bar{C}_{21} / \gamma$ at the BPM location just before the coupler can be calculated using the previous BPM (downstream) instead of the BPM upstream. This maintains the region between the two BPMs to be coupler free and the calculation of $\bar{C}_{21} / \gamma$ exact.

To investigate the effect of noise in BPMs, different levels of Gaussian noise were introduced into turn-byturn tracking data used to compute $\overline{\mathbf{C}}$ matrix. Figure 5 shows the normalized $\mathrm{rms}$ of the difference for $|C| / \gamma^{2}$ and $\overline{\mathbf{C}}$ matrix elements between model and calculated values as a function of $\sigma_{\text {noise }} /$ signal amplitude. It is clear that noise deteriorates the measurements. We observe that $\bar{C}_{12} / \gamma$ is more robust against noise than the other elements of the $\mathbf{C}$ matrix. The discrepancy of the effect of noise between $\bar{C}_{12} / \gamma$ and $\bar{C}_{22}$ can be attributed to the calculation of $\Delta \psi_{b, a}$ and is under study. $\bar{C}_{21} / \gamma$ inherently has large errors since Eq. (17) is exact only in a coupler free region. This can be improved by the choice of appropriate BPMs for the calculation as explained above. It was shown that the choice of number of turns in tracking affects the computation of $\overline{\mathbf{C}}$ matrix due to the periodicity effect caused by the number of significant digits in the tune [3]. In real data $\bar{C}_{22}$ unlike $\bar{C}_{12} / \gamma$ were shown to be susceptible to large errors due to signal "leaks" in BPMs and their corresponding electronics $[1,10]$.

\section{Calculation of $|\overline{\mathbf{C}}| / \boldsymbol{\gamma}^{\mathbf{2}}$ for RHIC lattice}

Single-particle tracking using RHIC lattice was performed to verify the applicability of this approach for a more realistic operating accelerator with several coupling 


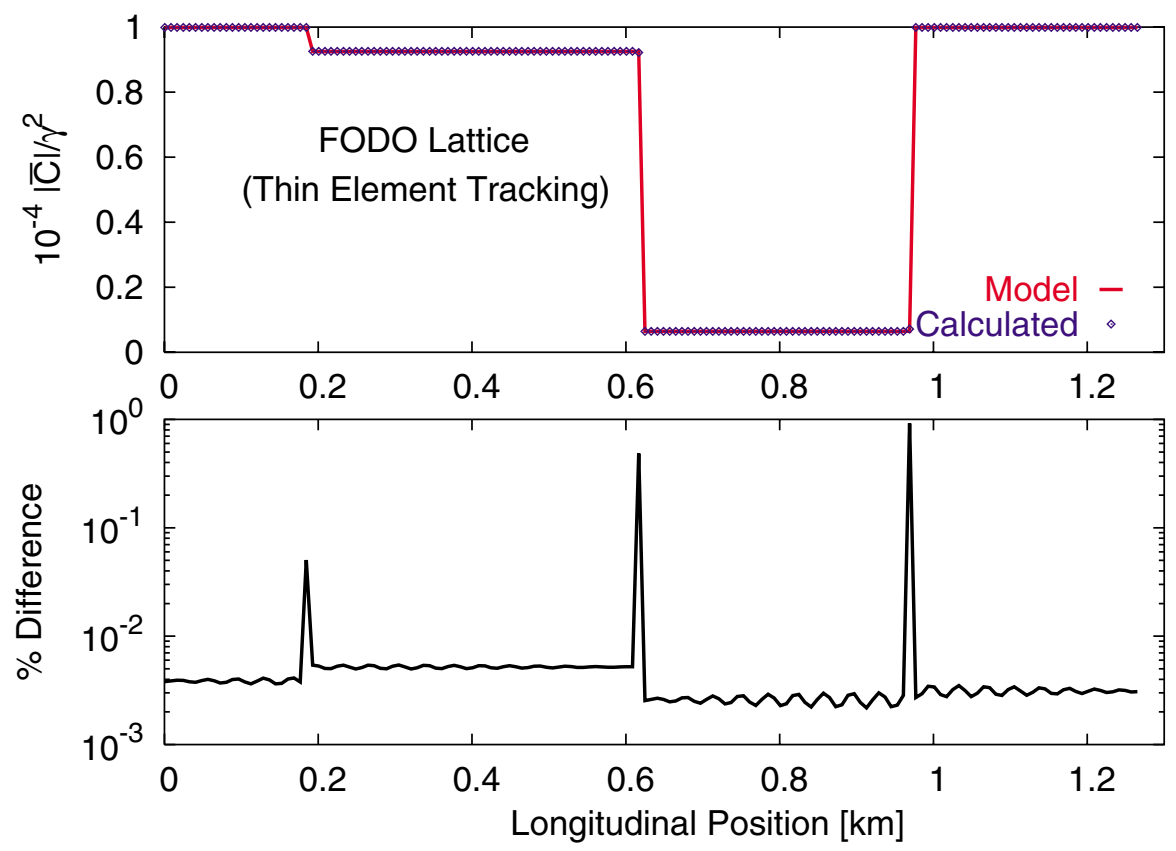

FIG. 4. (Color) Top: Comparison of $|C| / \gamma^{2}$ between MAD-X model and SVD computed values from tracking data. Bottom: Difference between model and calculated values of $|C| / \gamma^{2}$. Horizontal and vertical tunes are $Q_{x}=18.226$ and $Q_{y}=17.257$, respectively.

sources. RHIC consists of two threefold symmetric rings with six interaction regions. Each arc is made of 11 FODO cells with $80^{\circ}$ phase advance, and interaction regions consist of almost the same FODO cells without the dipoles [14]. A model of RHIC containing realistic but uncorrected errors is used to track a single particle for 2000 turns with $Q_{x}=28.266$ and $Q_{y}=29.212$. The $\Delta Q_{\min }$ for this lattice is $4.37 \times 10^{-2}$. Figure 6 shows a calculation of $|\overline{\mathbf{C}}| / \gamma^{2}$ from turn-by-turn data compared to model values from MAD-X. One can clearly see that the coupling sources are quite strong and mainly located in interaction regions. The agreement between model and calculated values of $|\overline{\mathbf{C}}| / \gamma^{2}$ is better in the arcs than in the interaction regions (IRs). The larger discrepancies arising in the IRs are due to the

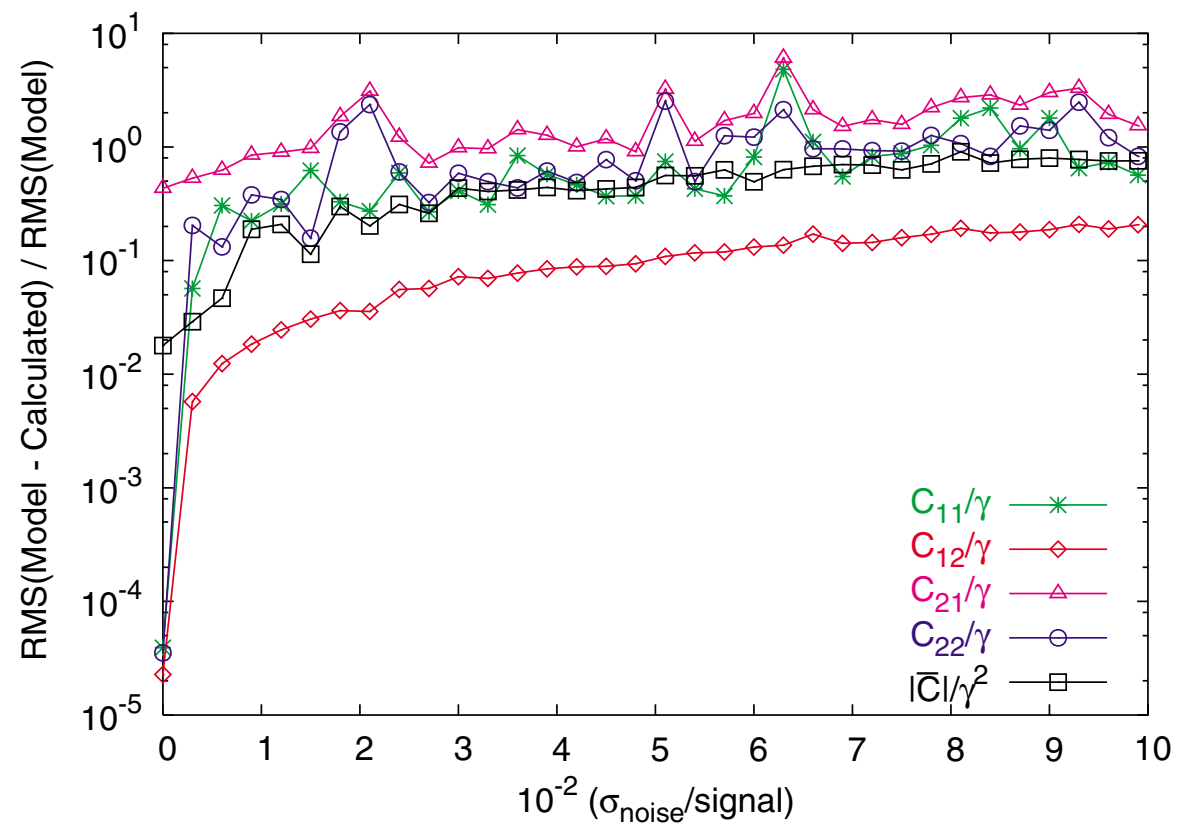

FIG. 5. (Color) Normalized rms of the difference of $|C| / \gamma^{2}$ between MAD-X model and SVD computed values for increasing amount of Gaussian noise in turn-by-turn data. Horizontal and vertical tunes are $Q_{x}=18.226$ and $Q_{y}=17.257$, respectively. 


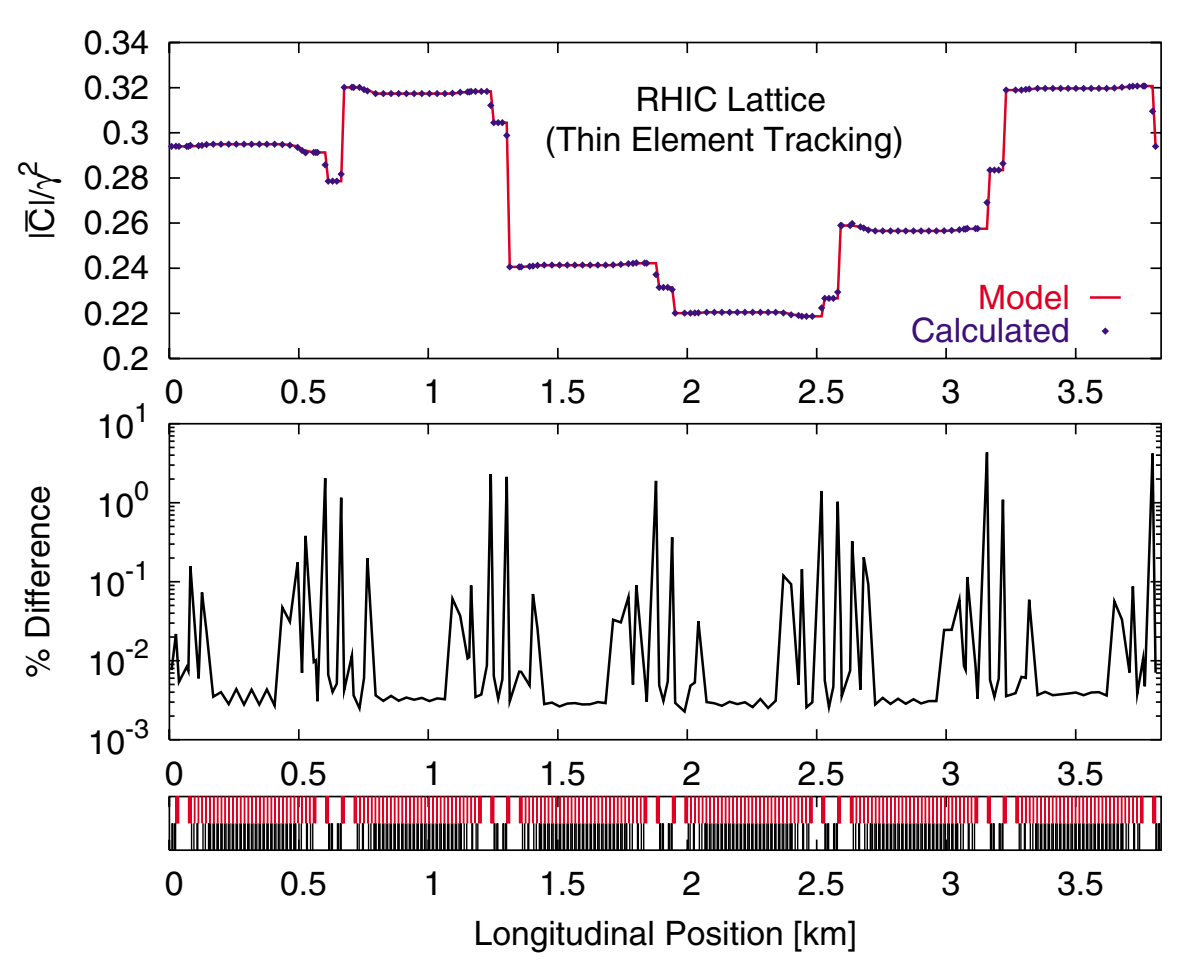

FIG. 6. (Color) Top: Comparison of $|\overline{\mathbf{C}}| / \gamma^{2}$ between MAD-X model and SVD computed values from tracking data. Middle: Difference between model and calculated values of $|\overline{\mathbf{C}}| / \gamma^{2}$. Bottom: A representation of the lattice (dipoles in black and quadrupoles in red) is shown in the bottom graph. The horizontal and vertical tunes were $Q_{x}=28.266$ and $Q_{y}=29.212$, respectively, and $\Delta Q_{\text {min }}=$ $4.37 \times 10^{-2}$.

presence of strong coupling sources. However, even in a complicated lattice like RHIC, the sources are clearly identified and all errors are below 5\%.

For the above simulation, we assumed dual-plane BPMs in the lattice. However, this is not true for RHIC and most operating hadron colliders. RHIC consists of 160 BPMs per plane per ring: 72 dual-plane BPMs distributed through the IRs and 176 single-plane BPMs distributed in the arcs capable of acquiring 1024 turns. The single-plane BPMs in RHIC are confined to the arc regions where coupling sources are minimal. A more sophisticated algorithm to estimate $|\overline{\mathbf{C}}| / \gamma^{2}$ with lattices consisting of both single and double plane BPMs is under investigation. In real accelerators, many BPMs routinely fail resulting in unreliable data. It has been shown that preprocessing of BPM data is usually effective in removing faulty BPMs and maintaining data integrity [15] to obtain reliable measurements.

\section{Calculation of skew quadrupole strengths}

In $[16,17]$ a method to obtain multipolar strengths from the measurement of RDTs was proposed. Skew quadrupole strengths are equivalently obtained from the measurement of the $\overline{\mathbf{C}}$ matrix by use of the above relations. According to Fig. 7 we assume that only one skew quadrupole of integrated strength $k$ exists between the two BPMs where the $\overline{\mathbf{C}}$ matrices have been measured. From [16,17] the integrated strength $\bar{k}$ is given by

$$
\begin{aligned}
\bar{k}= & -4 e^{-i\left(\phi_{x}^{\text {skew }}-\phi_{y}^{\text {skew }}\right)}\left(f_{1001}^{(l)} e^{i\left(\phi_{x}^{l}-\phi_{y}^{l}\right)}\right. \\
& \left.-f_{1001}^{(l-1)} e^{i\left(\phi_{x}^{l-1}-\phi_{y}^{l-1}\right)}\right)
\end{aligned}
$$

with $\bar{k}=k \sqrt{\beta_{x}^{\text {skew }} \beta_{y}^{\text {skew }}}$. Here $k$ is the strength of coupler, $\beta_{x, y}^{\text {skew }}$ and $\phi_{x, y}^{\text {skew }}$ are the Twiss functions at the location of the skew quadrupole, $\phi_{x, y}^{l}$ and $\phi_{x, y}^{l-1}$ are the betatron phases at the $l$ th and $(l-1)$ th BPMs, respectively, and $f_{1001}^{(l)}$ and $f_{1001}^{(l-1)}$ are the corresponding RDTs. These terms are given by Eq. (9) as a function of the measured $\overline{\mathbf{C}}$ matrix. The RDT $f_{1010}$ can also be used leading to a similar equation. It is also interesting to relate the change of the determinant of

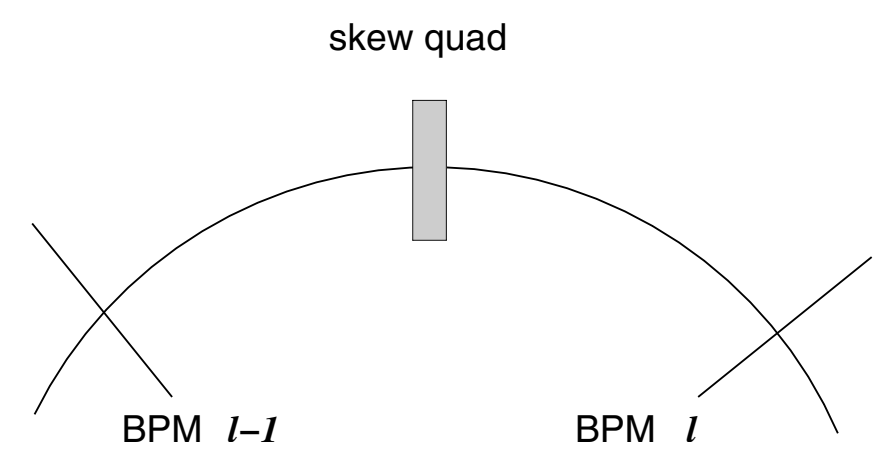

FIG. 7. Schematic view of a skew quad and the neighbor BPMs. 
$\overline{\mathbf{C}}$ to the strength of the skew quadrupole. By manipulating the above expressions (see alternate derivation in Sec. 2 of the appendix),

$$
\bar{k}=-\frac{1}{\chi}\left(\frac{\left|C^{(l)}\right|}{\gamma^{(l)^{2}}}-\frac{\left|C^{(l-1)}\right|}{\gamma^{(l-1)^{2}}}\right),
$$

where $\chi$ is given by

$$
\begin{aligned}
\chi= & \frac{1}{\gamma^{(l)}}\left(-\sin \delta \phi_{x} \sin \delta \phi_{y} \bar{C}_{21}^{(l)}+\cos \delta \phi_{x} \cos \delta \phi_{y} \bar{C}_{12}^{(l)}\right. \\
& \left.+\sin \delta \phi_{x} \cos \delta \phi_{y} \bar{C}_{22}^{(l)}-\cos \delta \phi_{x} \sin \delta \phi_{y} \bar{C}_{11}^{(l)}\right),
\end{aligned}
$$

where $\delta \phi_{x, y}=\phi_{x, y}^{\text {skew }}-\phi_{x, y}^{l}$ are the phase advances between the skew quadrupole and the second location of observation. Using Eq. (A4), Eq. (24) can also be expressed as

$$
\chi=\frac{C_{12}^{\text {skew }}}{\gamma^{l}} .
$$

To determine the applicability of the above expressions, a simulation using strongly coupled RHIC lattice is performed. The $\Delta Q_{\min }$ for this lattice is $4.37 \times 10^{-2}$. Figure 8 shows skew quadrupole strengths determined from Eq. (23) for the RHIC lattice. The presence of large coupling sources leads to relative errors of calculated strengths in the $20 \%$ level. This is due to the fact that these expressions are first order approximations and deviate with large coupling as illustrated in Sec. II D 2.

\section{CLOSEST TUNE APPROACH}

The following expression relating the closest tune approach and the determinant of $\overline{\mathbf{C}}$ is given in $[5,6]$,

$$
\Delta Q_{\min }=\frac{2 \gamma\left(\cos 2 \pi Q_{x}-\cos 2 \pi Q_{y}\right)}{\pi\left(\sin 2 \pi Q_{x}+\sin 2 \pi Q_{y}\right)} \sqrt{|\overline{\mathbf{C}}|} .
$$

This equation cannot hold true in general since its left-hand side is invariant around the ring but the right-hand side is not, as explained in Sec. II C. Only close to the difference resonance the determinant of $\overline{\mathbf{C}}$ tends to be invariant and Eq. (26) is considered to be a good approximation. Under this assumption the closest tune approach can also be related to the resonance terms,

$$
\begin{aligned}
\Delta Q_{\min }= & \frac{\cos 2 \pi Q_{x}-\cos 2 \pi Q_{y}}{\pi\left(\sin 2 \pi Q_{x}+\sin 2 \pi Q_{y}\right)} \\
& \times\left(\frac{4 \sqrt{\left|f_{1001}\right|^{2}-\left|f_{1010}\right|^{2}}}{1+4\left(\left|f_{1001}\right|^{2}-\left|f_{1010}\right|^{2}\right)}\right),
\end{aligned}
$$

and since we assume the tunes to be close to the difference resonance, the approximation $\left|f_{1001}\right| \gg\left|f_{1010}\right|$ might also be used [5,6]. A computer simulation is performed to investigate the validity of Eqs. (26) and (27). The same FODO lattice with three skew quadrupoles were used with horizontal and vertical tunes $Q_{x}=18.226$ and $Q_{y}=$ 17.239, respectively. Since $|\overline{\mathbf{C}}|$ varies around the ring,

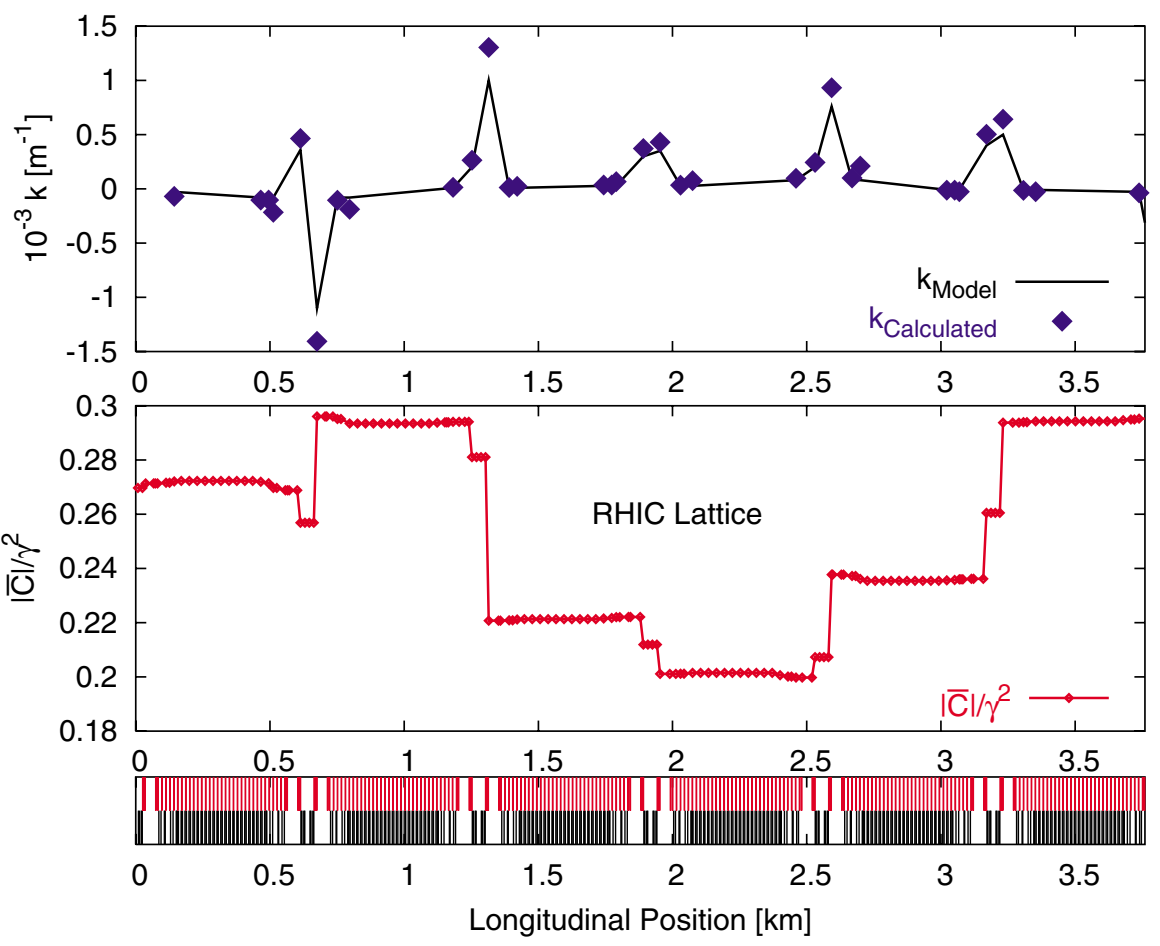

FIG. 8. (Color) Top: Skew quadrupole strengths calculated from RDTs and $\overline{\mathbf{C}}$ matrix are compared to MAD-X model values. Note that RDTs are calculated from $\overline{\mathbf{C}}$ matrix using Eqs. (9) and (10). Middle: $|\overline{\mathbf{C}}| / \gamma^{2}$ is plotted as a function longitudinal position. A representation of the lattice (dipoles in black and quadrupoles in red) is shown in the bottom graph. The horizontal and vertical tunes are $Q_{x}=28.266$ and $Q_{y}=29.212$, respectively, and $\Delta Q_{\min }=4.37 \times 10^{-2}$. 

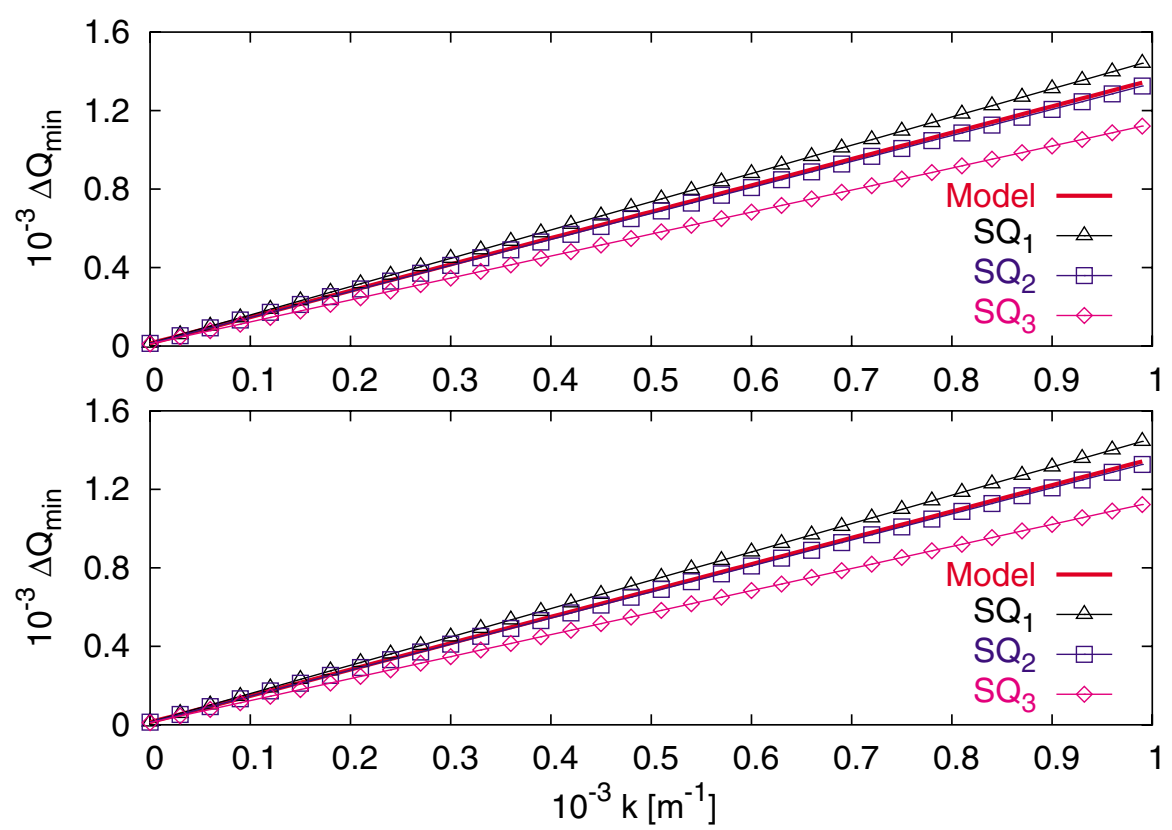

FIG. 9. (Color) Top: $\Delta Q_{\min }$ calculated using the three different values of RDTs at respective locations of skew quadrupoles as a function of their strength. Bottom: $\Delta Q_{\min }$ calculated using three different values of $|\overline{\mathbf{C}}|$ at respective locations of skew quadrupoles as a function of their strength $\left(Q_{x}=18.226, Q_{y}=17.239\right)$.

$\Delta Q_{\min }$ is calculated with the three values of $|\overline{\mathbf{C}}|$ at the three locations of skew quadrupoles and plotted as a function of increasing skew quadrupole strengths as shown in Fig. 9. We observe a dispersion in the $\Delta Q_{\text {min }}$ curves depending on the choice of the $|\overline{\mathbf{C}}|$ value used to calculate $\Delta Q_{\min }$. If the tunes are closer to the coupling resonance, the relative longitudinal variation in $|\overline{\mathbf{C}}|$ around the ring is smaller, hence reducing this dispersion.

\section{CONCLUSIONS}

Direct relations are established between the coupling matrix and the RDTs. This allows reinterpretation of the coupling matrix in terms of resonances and using results from both formalisms indistinctly. Numerical simulations are carried out to confirm these relations and explore their scope of application to real accelerators. The determinant of $\overline{\mathbf{C}}$ and the RDTs $\left(f_{1001}\right)$ have been demonstrated to exhibit distinct behavior that unambiguously reveals the region of the coupling sources.

A new approach to compute the full $\overline{\mathbf{C}}$ matrix and hence the determinant from turn-by-turn data is presented and comparison to model shows excellent agreement. An approach to extract the skew quadrupole strengths previously using RDTs is also extended to $\overline{\mathbf{C}}$ matrix. The applicability of the expression for $\Delta Q_{\min }$ from the $|\overline{\mathbf{C}}|$ has been discussed.

\section{ACKNOWLEDGMENTS}

The authors thank S. Peggs, W. Fischer, and Y. Luo for useful discussions and comments on the manuscript. The authors would like to acknowledge C.X. Wang for useful discussions on SVD based coupling analysis. The authors also thank F. Schmidt and A. Verdier for their help in upgrading the MAD-X tracking module. This work was performed under the auspices of the U.S. Department of Energy.

\section{APPENDIX: PROPAGATION OF THE $\overline{\mathrm{C}}$ MATRIX}

In a coupler free region $\overline{\mathbf{C}}$ matrix is simply propagated by an arbitrary phase advance in both modes, which is given by [9]

$$
\overline{\mathbf{C}}_{\mathbf{2}}=\mathbf{R}_{x}\left(\phi_{x}\right) \overline{\mathbf{C}}_{\mathbf{1}} \mathbf{R}_{y}^{-1}\left(\phi_{y}\right)
$$

where

$$
\mathbf{R}_{\mathbf{x}, \mathbf{y}}=\left(\begin{array}{cc}
\cos \phi_{x, y} & \sin \phi_{x, y} \\
-\sin \phi_{x, y} & \cos \phi_{x, y}
\end{array}\right) .
$$

If coupling is small and couplers modeled as thin skew quadrupoles, the $\overline{\mathbf{C}}$ is propagated to first order given by [10]

$$
\overline{\mathbf{C}}_{2}=\overline{\mathbf{C}}_{1}-\overline{\mathbf{k}},
$$

where

$$
\overline{\mathbf{k}}=\left(\begin{array}{cc}
0 & 0 \\
\bar{k} & 0
\end{array}\right)
$$

with $\bar{k}=\sqrt{\beta_{a}^{\text {skew }} \beta_{b}^{\text {skew }}} k$, and $k$ is the strength of the coupler. Here $\gamma$ is assumed to be 1 . 


\section{1. $\bar{C}_{21}$ in coupler free region}

Using Eq. (A1), $\overline{\mathbf{C}}$ matrix elements at two locations are related in terms of the phase advance alone, which is expressed as

$$
\left(\begin{array}{c}
C_{11}^{(2)} \\
C_{12}^{(2)} \\
C_{21}^{(2)} \\
C_{22}^{(2)}
\end{array}\right)=\left(\begin{array}{cccc}
c \phi_{x} c \phi_{y} & c \phi_{x} s \phi_{y} & s \phi_{x} c \phi_{y} & s \phi_{x} s \phi_{y} \\
-c \phi_{x} s \phi_{y} & c \phi_{x} c \phi_{y} & -s \phi_{x} s \phi_{y} & s \phi_{x} c \phi_{y} \\
-s \phi_{x} c \phi_{y} & -s \phi_{x} s \phi_{y} & c \phi_{x} c \phi_{y} & c \phi_{x} s \phi_{y} \\
s \phi_{x} s \phi_{y} & -c \phi_{x} c \phi_{y} & -c \phi_{x} s \phi_{y} & c \phi_{x} c \phi_{y}
\end{array}\right)\left(\begin{array}{c}
C_{11}^{(1)} \\
C_{12}^{(1)} \\
C_{21}^{(1)} \\
C_{22}^{(1)}
\end{array}\right),
$$

where $c \phi=\cos (\phi)$ and $s \phi=\sin (\phi)$. Given two BPM locations at which turn-by-turn data is recorded, $\bar{C}_{12} / \gamma$, $\bar{C}_{11} / \gamma$, and $\bar{C}_{22} / \gamma$ are calculated as illustrated in Sec. III A. The phase advances between the two locations can also be determined using SVD techniques from the same turn-byturn data [13]. Rearranging the second row of Eq. (A4), $\bar{C}_{21} / \gamma$ is exactly calculated in a coupler free region which is given by

$$
\begin{aligned}
\bar{C}_{21}^{(1)}= & \left(-\bar{C}_{11}^{(1)} \cos \phi_{a} \sin \phi_{b}+\bar{C}_{12}^{(1)} \cos \phi_{a} \sin \phi_{b}\right. \\
& \left.+\bar{C}_{22}^{(1)} \sin \phi_{a} \cos \phi_{b}-\bar{C}_{12}^{(2)}\right) /\left(\sin \phi_{a} \sin \phi_{b}\right) .
\end{aligned}
$$

\section{Skew quadrupole strength from two BPMs}

Using Eqs. (A1) and (A2), $\overline{\mathbf{C}}$ matrix is propagated between two observation points with one skew quadrupole between them given by

$$
\begin{aligned}
\overline{\mathbf{C}}_{\mathbf{2}}= & \mathbf{R}_{x}\left(\phi_{x}^{\text {skew }+l}\right)\left[\mathbf{R}_{x}\left(\phi_{x}^{\text {skew }-l}\right) \overline{\mathbf{C}}_{\mathbf{1}} \mathbf{R}_{y}^{-1}\left(\phi_{y}^{\text {skew }-l}\right)\right. \\
& -\overline{\mathbf{k}}] \mathbf{R}_{y}^{-1}\left(\phi_{y}^{\text {skew }+l}\right),
\end{aligned}
$$

where $\phi_{x, y}^{\text {skew } \mp l}$ are the phase advances between the skew quadrupole and locations (1) and (2), respectively. Determinants are distributive $(|\mathbf{A B}|=|\mathbf{A}| \times|\mathbf{B}|)$, therefore

$$
\left|\overline{\mathbf{C}}_{\mathbf{2}}\right|=\left|\mathbf{R}_{x}\left(\phi_{x}^{\text {skew }-l}\right) \overline{\mathbf{C}}_{\mathbf{1}} \mathbf{R}_{y}^{-1}\left(\phi_{y}^{\text {skew }-l}\right)-\overline{\mathbf{k}}\right|,
$$

since $\left|\mathbf{R}_{x}\left(\phi_{x, y}\right)\right|=1$. Using Eqs. (A4) and (A7) $\bar{k}$ is expressed as

$$
\bar{k}=-\frac{\left|C^{(2)}\right|-\left|C^{(1)}\right|}{C_{12}^{\text {skew }}},
$$

which is equivalent to Eq. (23) derived from RDTs given that $\gamma=1$.
[1] P. Bagley and D. Rubin, in Proceedings of the 1997 Particle Accelerator Conference, Chicago (IEEE, Piscataway, NJ, 1989), p. 874.

[2] D. Sagan, R. Meller, R. Littauer, and D. Rubin, Phys. Rev. ST Accel. Beams 3, 092801 (2000).

[3] C.X. Wang and R. Calaga, in Proceedings of the European Particle Accelerator Conference, Lucerne, Switzerland, 2004 (EPAC 2004), http://accelconf.web.cern.ch/accelconf/e04/, p. 1470.

[4] R. Tomás, Ph.D. thesis, Universitat de Valencia, 2002.

[5] L. Schachinger and R. Talman, Manual for the Program TEAPOT, Noninteractive FORTRAN Version," Appendix G, 1996 (unpublished).

[6] W. Fischer, Phys. Rev. ST Accel. Beams 6, 062801 (2003).

[7] R. Bartolini and F. Schmidt, Part. Accel. 59, 93 (1998).

[8] D. Edwards and L. Teng, IEEE Trans. Nucl. Sci. 20, 3 (1973).

[9] D. Sagan and D. Rubin, Phys. Rev. ST Accel. Beams 2, 074001 (1999).

[10] D. Sagan, Phys. Rev. ST Accel. Beams 3, 102801 (2000).

[11] F. Schmidt, MAD-X User's Guide, Version 2.12 (CERN, Geneva, 2003).

[12] C.X. Wang, Phys. Rev. ST Accel. Beams 7, 114001 (2004).

[13] J. Irwin, C. X. Wang, Y. T. Yan, K. L. F. Bane, Y. Cai, F.-J. Decker, M. G. Minty, G. V. Stupakov, and F. Zimmermann, Phys. Rev. Lett. 82, 1684 (1999).

[14] M. Harrison, S .Peggs, and T. Roser, Annu. Rev. Nucl. Part. Sci. 52, 425 (2002).

[15] R. Calaga and R. Tomás, Phys. Rev. ST Accel. Beams 7, 042801 (2004).

[16] R. Tomás, M. Bai, W. Fischer, A. Franchi, and G. Rumolo, in Proceedings of the European Particle Accelerator Conference, Lucerne, Switzerland, 2004 (EPAC 2004) (Ref. [3]).

[17] A. Franchi, T. Beier, M. Kirk, P. Moritz, G. Rumolo, and R. Tomás, in Proceedings of the European Particle Accelerator Conference, Lucerne, Switzerland, 2004 (EPAC 2004) (Ref. [3]). 